\title{
Free-space optical channel performance under atmospheric losses using orthogonal frequency division multiplexing
}

\author{
Vinoth Kumar Mani, Vinod Kumar \\ Department of Electronics and Communication Engineering, SRM Institute of Science and Technology, Ghaziabad, India
}

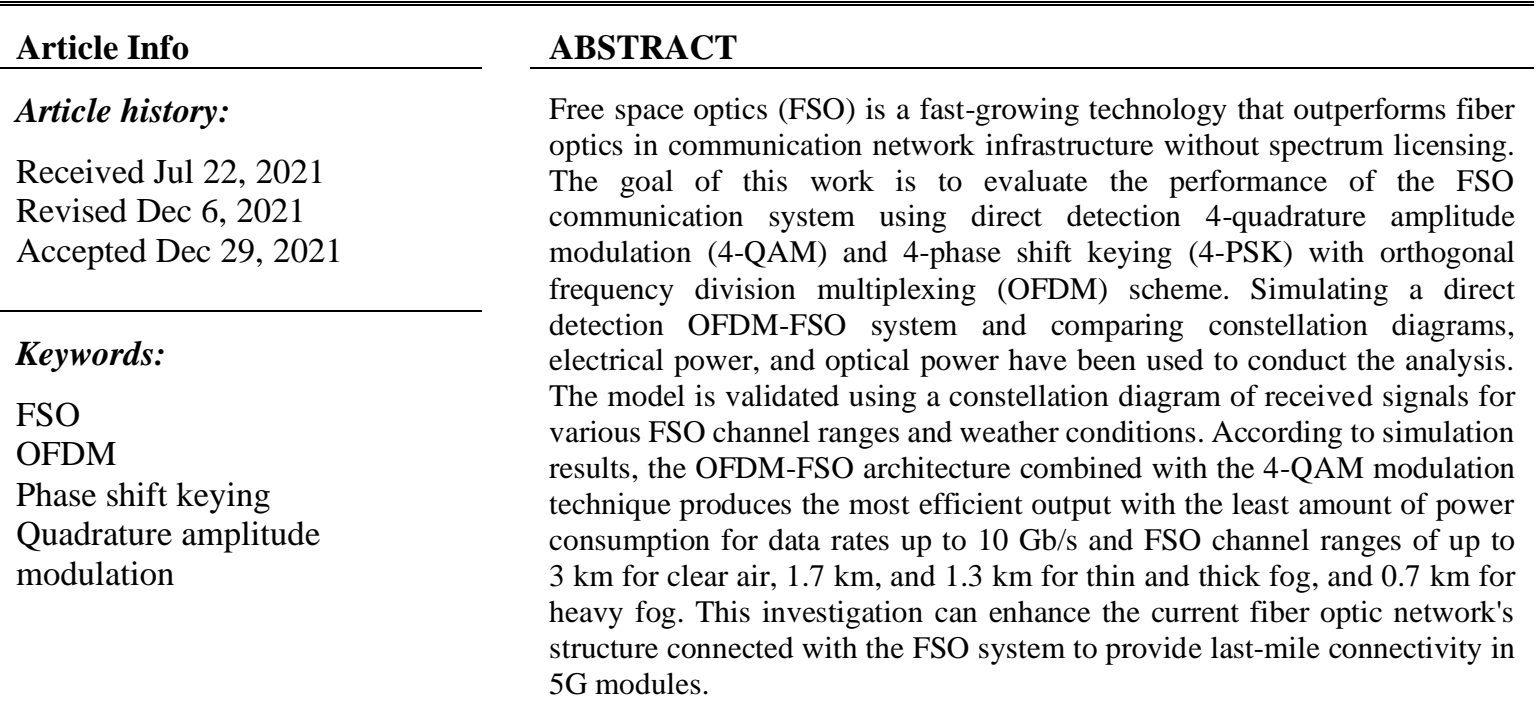

This is an open access article under the $\underline{C C B Y-S A}$ license.

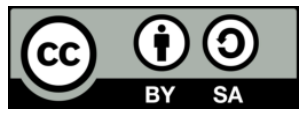

\section{Corresponding Author:}

Vinod Kumar

Department of Electronics and Communication Engineering, SRM Institute of Science and Technology

Delhi NCR Campus, Ghaziabad, India

Email: vinodkur1@srmist.edu.in

\section{INTRODUCTION}

The recent growth in telecommunications and the widespread use of cellular systems such as smartphones, satellite communication, smart sensors, and surveillance systems have increased the complexity of algorithms used in the latest generation of technologies, causing them to reach their limits. Many studies have been conducted with fiber optic channels to deal with the problems of high data rate transmission at high speed [1], [2]. The fiber communication system has the advantages of noise immunity, free from electromagnetic interference, secure systems. Fiber bending loss and cost are two issues that arise when installing fiber optic cables across buildings in a metropolitan area [3], [4]. Free space optical (FSO) systems have gained much interest recently as a way to avoid bending loss and achieve last-mile communication. Several types of research have been conducted to improve transmission rates using free space and unlicensed bandwidths. Over the last several years, FSO communication has grown in popularity as an alternative technology [5]. Atmospheric turbulence and various weather conditions cause attenuation of the broadcast signal across free space, reducing the FSO channel's possibilities.

Because its bandwidth in FSO is license-free, cost-efficient, low power consumption, more excellent immunity, and highly secured when compared to radiofrequency (RF) systems, it can enable transmission ranges up to $1.2 \mathrm{Tbps}$ [6]. The impacts of air turbulence, channel misalignment, changes in weather conditions, 
and the parameters describing these effects are significant concerns for signal attenuation while designing an FSO channel. Few techniques to overcome these challenges are: i) hybrid FSO-RF system, ii) hybrid FSOfiber system, and iii) multiple-input multiple-output (MIMO) system along with the implementation of advanced modulation schemes [5]. Digital modulation schemes are analyzed based on energy efficiency requirement, targeted spectral efficiency, and direct or coherent detection system. In this work, the direct detection scheme is implemented using the 4-quadrature amplitude modulation (4-QAM) and 4-phase shift keying (4-PSK) orthogonal frequency division multiplexing (OFDM) schemes. Constellation diagrams are presented for various FSO channel lengths, including attenuation for haze and fog weather conditions. Previous research works on digital modulation schemes analyzed to mitigate atmospheric turbulence are on-off keying (OOK) [7], binary phase-shift keying (BPSK) [8], pulse position, and multiple pulse position modulations (PPM and MPPM) [9], [10], pulse width modulation (PWM) [11]. Forward error correction techniques (FEC), for example, reed solomon concatenated reed solomon, and turbo codes [12], [13], and selection combining technique are a few promising techniques to mitigate atmospheric turbulence [14]. Spatial modulation increases the spectral efficiency of FSO communication [15]. Signal loss related to atmospheric attenuation and pointing errors due to FSO channel misalignment causes propagated signal strength to deteriorate during transmission, lowering system efficiency. These issues can be overcome by relay methods MIMO systems [16]. MIMO systems were modeled and evaluated in the earlier study. The findings of bit error rate (BER), quality factor (Q-factor), and eye diagram indicated a significant reduction in atmospheric attenuation. 5G systems can achieve self-configuration, context awareness, and cellular aggregation with deep learning models. Deep learning (DL) algorithms could efficiently recover the transmitted data, thus allowing FSO in turbulent channels to be used without prior knowledge. Various papers have examined the scintillation of the optical beam, which is the primary cause of pointing errors in FSO systems because of the line-of-sight nature. DL and artificial intelligence (AI) algorithms provide the most economical way to overcome this problem [17], [18], and these algorithms are applied in broad applications like self-driving cars, visual recognition, healthcare [19], [20]. Different deep learning models can be applied to different strengths of FSO turbulent channels to detect OOK modulated signals [21]. A machine-learning-based methodology was presented for improving future optical wireless communication systems from existing fiber-based networks to cognitive networks based on fiber-based learning that provides cognitive capabilities at the physical layer [22].

The goal of this study is to model and simulate an OFDM-FSO communication system with 4-QAM, 4-PSK, and to analyze system performance under various weather conditions such as clear air, haze, fog, and various FSO wireless channel ranges. The proposed system was modeled and simulated using optisystem 17. At the transmitter side, the simulation model includes a low pass cosine roll-off filter (LP roll-off filter) and a LiNb-mach zehnder modulator (LiNb-MZM) as an electrical to an optical converter, FSO channel of gammagamma model and PIN photodiode, OFDM demodulation blocks at receiver. Simulated results are comparing constellations for various weather conditions at OFDM output. The proposed system's performance and reliability outcomes connect to the low pass filter's roll-off factor and the FSO channel's ranges.

\section{METHOD}

\subsection{OFDM system}

OFDM is a multicarrier modulation system where subcarriers signals are mutually orthogonal [23]. The principle of the OFDM technique involves a serial data stream that is converted into a parallel data stream for a longer duration. OFDM presents a large number of orthogonal subcarriers to transmit data across multiple parallel data streams. Each carrier is processed at a very low symbol rate using standard quadrature phase shift keying (QPSK), QAM, or a similar digital modulation technique. Direct and coherent detection are two different optical OFDM detection methods [24]. A photodiode at the receiver detects an optical signal in a direct detection scheme, and the received signal is demodulated. In contrast, a coherent detection system has used the optical mixing principle with a locally set oscillator at the receiver.

The optical signal from the transmitter is transmitted through single or multi-mode optical fibers, and free-space optical wireless channels are used; several electronics and optical processing are involved inbetween. A guard interval is placed after the inverse fast fourier transform (IFFT) module in the OFDM system to avoid overlapping signals. The digital data are converted to an analog signal in the next step. The receiver performs a similar process as the transmitter but an opposite function.

\subsection{FSO system}

FSO is a kind of unguided medium and line-of-sight technology that uses laser as an optical source to offer optical bandwidth connection. The signal propagates over free space such as outer space, air, and vacuum. In general, FSO can transmit up to $2.5 \mathrm{Gbps}$ of information signal (voice/video signals), and it operates between $780 \mathrm{~nm}$ to $1600 \mathrm{~nm}$ wavelength bands. Three components are there to define the FSO system are: i) transmitter 
aperture, ii) FSO transmission channel, and iii) receiver. A general block diagram of the FSO system is shown in Figure 1. Transmission of an optical signal via atmosphere obeys Beer-Lamberts Law, and in the FSO channel exists turbulent eddies such as smoke, gases, rain, fog, and variations in climatic temperature [25]. Typical FSO links range from $300 \mathrm{~m}$ to $5 \mathrm{~km}$. Depending on the speed and needed availability in free space, the link can be deployed as $8 \mathrm{~km}$ to $11 \mathrm{~km}$ [23].

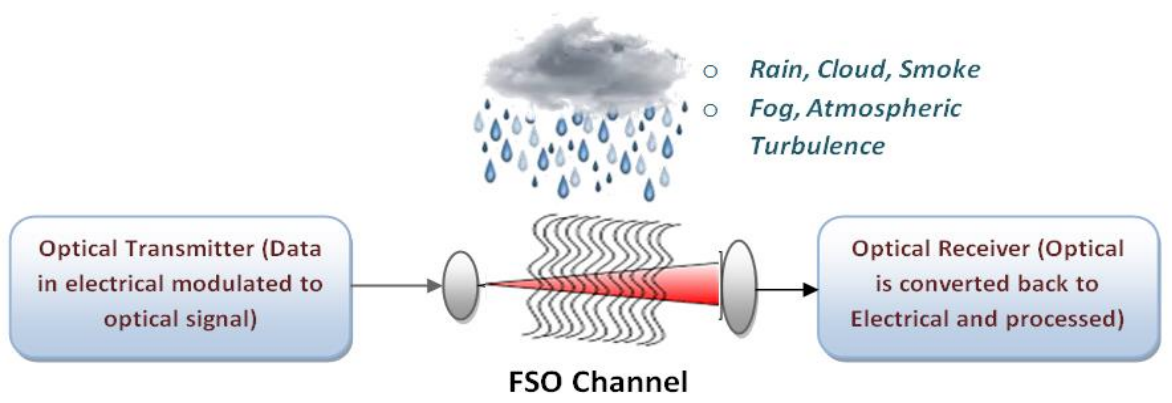

Figure 1. Free space optical system block diagram

FSO system is applicable in telecommunication and is considered a promising technology for 5G and 6G development, computer networking, communication among spacecraft, security, and military applications. FSO links are alternatives to fiber optical links to reduce bending losses in the metropolitan area where too many fiber bendings are implemented. The attenuation of the signal is a significant consideration in the FSO system. Atmospheric attenuation of laser power is defined by Beer-Lambert Law [26]:

$$
\tau=e^{-\sigma R}
$$

$\tau=$ Transmittance, $\sigma=$ Attenuation coefficient, $\sigma$ value is $0.1(0.43 \mathrm{~dB} / \mathrm{km})$ for clear air; $1(4.3 \mathrm{~dB} / \mathrm{km})$ for haze, $10(43 \mathrm{~dB} / \mathrm{km})$ for fog. The total scattering or the attenuation coefficient can be estimated by,

$$
\sigma=\frac{3.91}{V}\left(\frac{\lambda}{550 n m}\right)^{-q(v)}
$$

$\sigma=$ atmospheric attenuation co-efficient, $V=$ visibility (in $\mathrm{km}$ ), $\lambda=$ wavelength (in $\mathrm{nm}$ ), $q(v)=$ the size distribution of the scattering particles.

Table 1 illustrates the atmospheric losses $(\mathrm{dB} / \mathrm{km})$ calculated using as (2) as a function of visibility for $1550 \mathrm{~nm}$ [25]. This proposed model uses a continuous wave $(\mathrm{CW})$ laser with a wavelength of $1550 \mathrm{~nm}$. Attenuation in clear air $0.2 \mathrm{~dB} / \mathrm{km}$, haze $4.2 \mathrm{~dB} / \mathrm{km}$, thin fog $9.2 \mathrm{~dB} / \mathrm{km}$, and thick fog $22 \mathrm{~dB} / \mathrm{km}$ are used to analyze the system in this work.

Table 1. Atmospheric losses

\begin{tabular}{ccc}
\hline Visibility $(\mathrm{km})$ & Weather & $\mathrm{dB} / \mathrm{km}, 1550 \mathrm{~nm}$ \\
\hline 0.2 & FOG & 60 \\
0.5 & & 21 \\
2 & HAZE & 4 \\
4 & & 2 \\
10 & CLEAR & 0.4 \\
23 & & 0.2 \\
\hline
\end{tabular}

\subsection{Direct detection DD-OFDM-FSO proposed model}

Model of FSO communication system with OFDM-4 QAM scheme consists of OFDM modulator, electrical to an optical converter, wireless optical link, optical to the electrical converter and OFDM demodulator. Figure 2 depicts the block diagram of the proposed model. RF/optical spectrum analyzers, power meters (for both optical and electrical), and constellation visualize are used to visualize simulation results. 


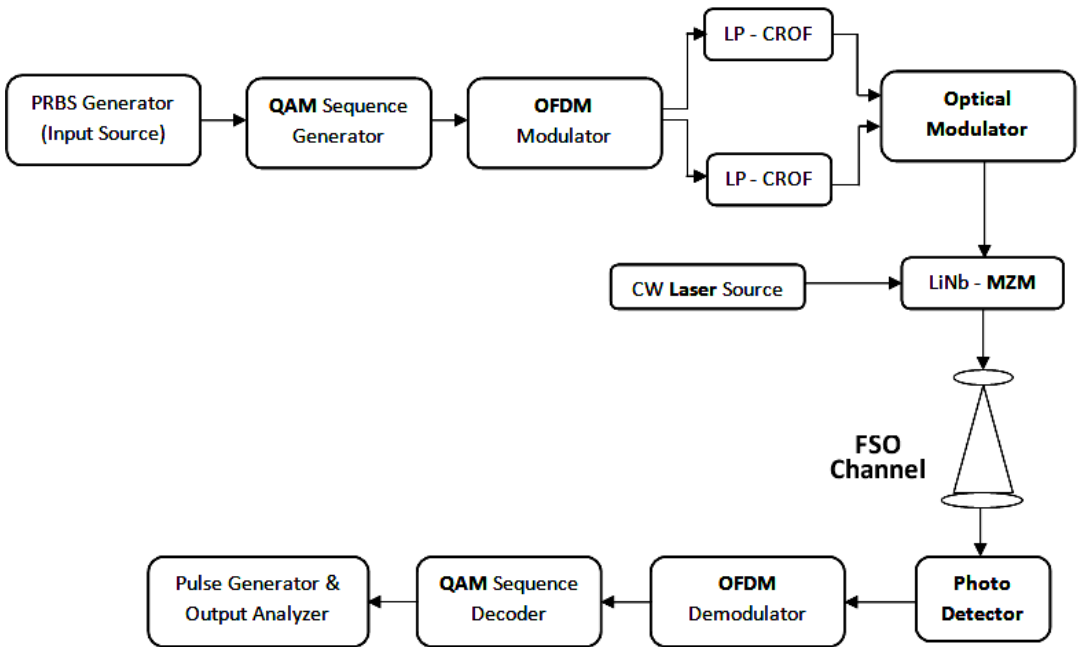

Figure 2. DD-OFDM-FSO simulation model

\subsubsection{OFDM transmitter}

The transmitter section consists of a pseudo-random bit sequence generator (PRBS) as a message generating module followed QAM sequence generator. 2 bits per symbol-4 QAM signal is coded as input to the OFDM modulator. OFDM modulator results in 512 subcarriers at 4-QAM and fast fourier transform (FFT) points of 1024. The subcarriers spacing is $1 / N T$ ( $N$-Number of carriers, $1 / T-$ Overall symbol rate). Several subcarriers are translated into points of IFFT. Cyclic prefix overcomes inter symbol/inter-channel interference. After the cyclic code, the parallel data are converted to series, and then the OFDM signal via quadrature modulator is applied to the external MZM modulator. Another input to MZM is light from the laser source. The laser source was used with $193.1 \mathrm{THz}$ and $4 \mathrm{dBm}$ power input parameters. The role of MZM is to modulate light intensity as per the sequence applied from the OFDM module. Optisystem component OFDM OS12 is used with I and Q output applied to low pass filters. 4-QAM OFDM FSO simulation model is shown in Figure 3.

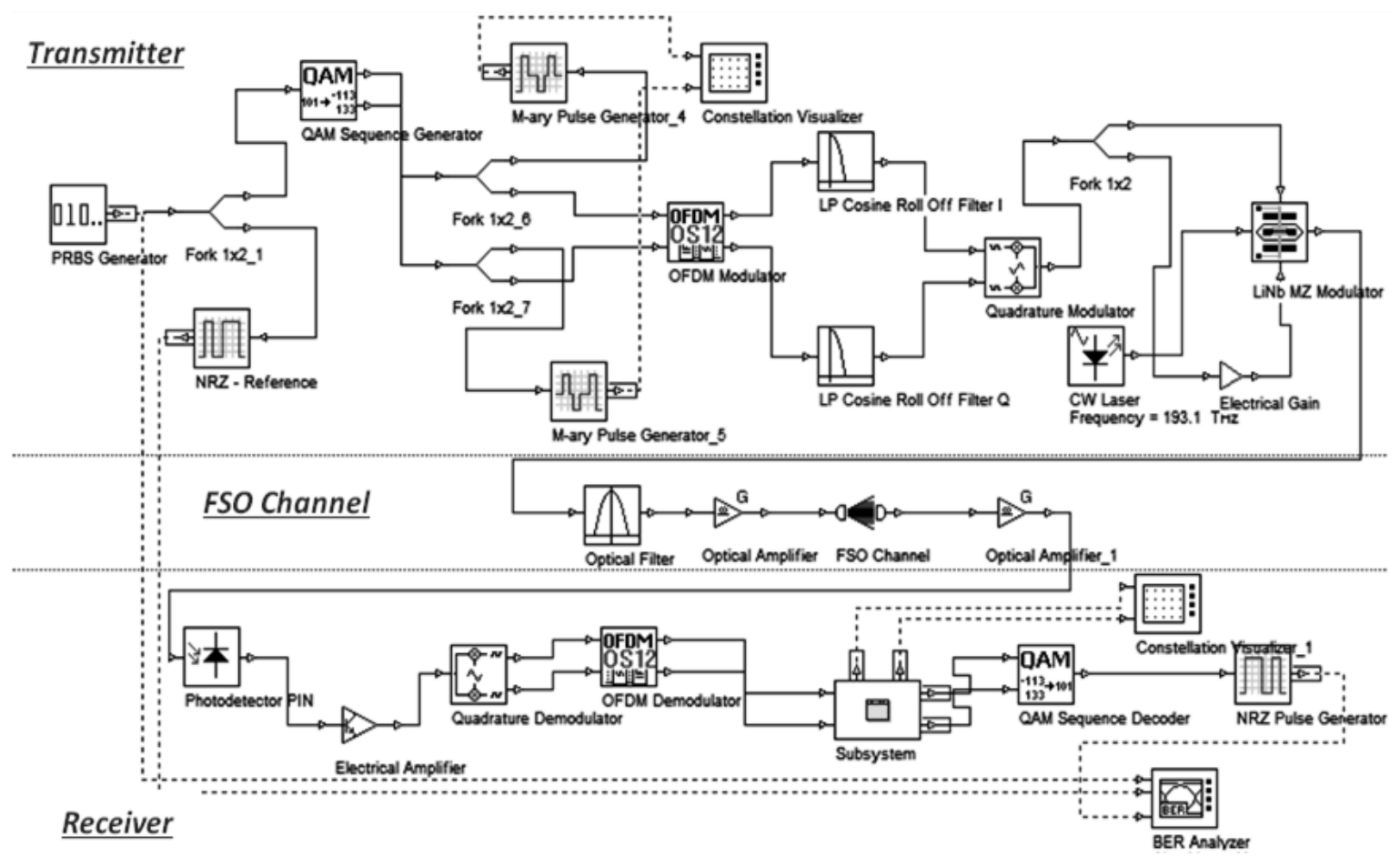

Figure 3. 4-QAM OFDM FSO system 
The roll-off factor value in the low pass filter (LP-CROF) is 0.2. Increasing roll-off factor value affects system performance. Low pass filters transfer function is developed by raised cosine-filter which is given by [27],

$$
H(f)=\left\{\begin{array}{rr}
\alpha & \left(|f|<f_{1}\right) \\
\beta & \left(f_{1} \leq|f|<f_{2}\right) \\
0 & f_{2}<|f|
\end{array}\right.
$$

where, $\beta=\sqrt{0.5 * \alpha^{2} *\left[1+\cos \left(\left(\frac{|f|-f_{1}}{r_{p} * \Delta f_{F W H M}}\right) * \pi\right)\right]}$

$\alpha=$ parameter insertion loss, $f_{c}=$ filter cutoff frequency, $r_{p}=$ roll-off factor, $\mathrm{f}_{1}=1-\mathrm{r}_{\mathrm{p}} \mathrm{f}_{\mathrm{c}} \quad\left(0 \leq \mathrm{r}_{\mathrm{p}} \leq 1\right)$, $\mathrm{f}_{2}=1+\mathrm{r}_{\mathrm{p}} \mathrm{f}_{\mathrm{c}} \quad\left(0 \leq \mathrm{r}_{\mathrm{p}} \leq 1\right)$.

Table 2 lists the parameters for the system model simulation. System parameters have been used to compare the performances of both 4-QAM and 4-PSK modulations with DD-OFDM techniques for the FSO system. FSO channel distance was varied from $0.5 \mathrm{~km}$ to $3 \mathrm{~km}$ for both modulation techniques and compared the received constellation points.

Table 2. Parameters used in the system model

\begin{tabular}{lc}
\hline \multicolumn{1}{c}{ Parameter } & Value \\
\hline Bit rate & $10 \mathrm{Gbps}$ \\
Sample rate & $40 \mathrm{Gbps}$ \\
Modulation scheme & $4 \mathrm{QAM}$ \\
Sample per bit & 4 \\
Number of samples & 65536 \\
Number of subcarriers & 512 \\
Quadrature modulator frequency & $7.5 \mathrm{GHz}$ \\
CW laser range & $193.1 \mathrm{THz}$ \\
LiNb MZ modulator extinction ratio & $30 \mathrm{~dB}$ \\
FSO Channel range & $0.5-3 \mathrm{~km}$ \\
\hline
\end{tabular}

\subsubsection{Optical link (FSO channel)}

The free-space optical link used in this model is Gamma-Gamma distribution. From weak to strong range of atmospheric turbulence conditions, the fading gain $I_{m n}$ is modeled by Gamma-Gamma distribution. Its probability distribution function is expressed as [28], [29]:

$$
\mathrm{f}_{\mathrm{I}}\left(\mathrm{I}_{\mathrm{mn}}\right)=\frac{2(\alpha \beta)^{\frac{\alpha+\beta}{2}}}{\Gamma(\alpha) \Gamma(\beta)} \mathrm{I}_{\mathrm{mn}}^{\frac{\alpha+\beta}{2}} 1 \mathrm{~K}_{\alpha-\beta}\left(2 \sqrt{\alpha \beta \mathrm{I}_{\mathrm{mn}}}\right)
$$

where $\Gamma(\alpha)$ is Gamma function, $K v(*)$ is the $v^{\text {th }}$ order modified Bessel function of the second kind [30], parameters $\alpha$ and $\beta$ both are calculated based on atmospheric conditions using the following expressions [27], [31],

$$
\alpha=\left[\exp \left(\frac{0.49 \chi^{2}}{\left(1+0.18 \mathrm{~d}^{2}+0.56 \chi^{\frac{12}{5}}\right)^{\frac{7}{6}}}\right)-1\right]^{-1}, \beta=\left[\exp \left(\frac{0.51 \chi^{2}\left(1+0.69 \chi^{\frac{12}{5}}\right)^{\frac{-5}{6}}}{\left(1+0.9 \mathrm{~d}^{2}+0.62 \mathrm{~d}^{2} \chi^{\frac{12}{5}}\right)^{\frac{7}{6}}}\right)-1\right]^{-1}
$$

where $\chi^{2}=0.5 C_{n}^{2} k^{(7 / 6)} L^{(11 / 6)}, d=\left(\frac{k D^{2}}{4 L}\right)^{1 / 2}, \mathrm{k}=2 \pi / \lambda, D=$ Diameter of the receiver lens aperture, $L=$ link distance in meters, $C_{n}^{2}$ is an altitude-dependent index of refractive structure parameter varying from $10^{-13} \mathrm{~m}^{-2 / 3}$ to $10^{-17} \mathrm{~m}^{-2 / 3}$ as strong to weak turbulence, respectively.

\subsubsection{Receiver}

Because the work is based on direct detection, a PIN photodetector transforms the incoming modulated light wave into an electrical signal. Then the electrical signal is amplified and demodulated. Demodulation is identical to modulation at the transmitter, but the concepts are reversed. The constellation diagram visualizers show the signal reception with errors and unmapped constellation points with noise, and power consumption is displayed in Table 3. 


\section{RESULTS AND DISCUSSION}

The proposed model's performance has been verified using optisystem 17. Simulated results are shown in Figures 4-6. Orthogonal frequency division multiplexing modulation baseband signal spectrum signal is shown in Figure 4(a) baseband data has been set $10 \mathrm{~Gb} / \mathrm{s}$, and the signal spectrum is more harmonic. Also, OFDM output modulated by 4 QAM digital technique has interval value up to $20 \mathrm{GHz}$. The spectrum has a higher amplitude of 28 $\mathrm{dBm}$. Low pass filter (LP-CROF) results in lower spectrum higher noise at $15 \mathrm{GHz}$ with roll-off factor value of 0.2 shown in Figure 4(b) increasing roll factor value broadens band transition filter (shown in Figure 4(c) roll-off factor value is 1), which affects adjacent frequency channels in case of multiple transmitters used. In this model roll-off factor of 0.2 is used for complete analysis. The LiNb MZ modulator modulates the optical signal with a maximum power value in its spectrum is up to $-10 \mathrm{dBm}$. FSO channel transmits the modulated optical signal. FSO link distance up to $3 \mathrm{~km}$ between transmitting and receiving aperture are tested with the attenuation of $0.2 \mathrm{~dB} / \mathrm{km}$ for clear weather conditions and $4.2 \mathrm{~dB} / \mathrm{km}, 9.2 \mathrm{~dB} / \mathrm{km}$, and $22 \mathrm{~dB} / \mathrm{km}$ for haze and fog weather conditions. Optical filtering and amplifier are used with FSO channel link helped to filter decided band and increase power. At the receiver, the optical signal is converted to an electrical signal. Because the direct detection (DD) technique is used in this research, a PIN photodiode is used to detect the optical signal. Figure 4(d) depicts the received signal spectrum before electrical amplification. Green waves reveal some noise in the output of the DD scheme.

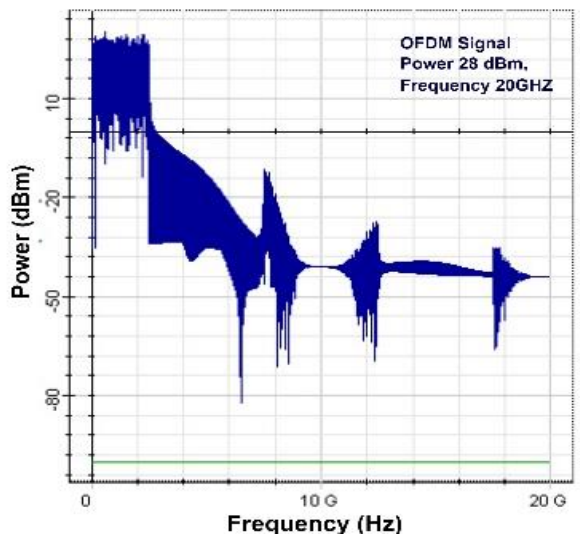

(a)

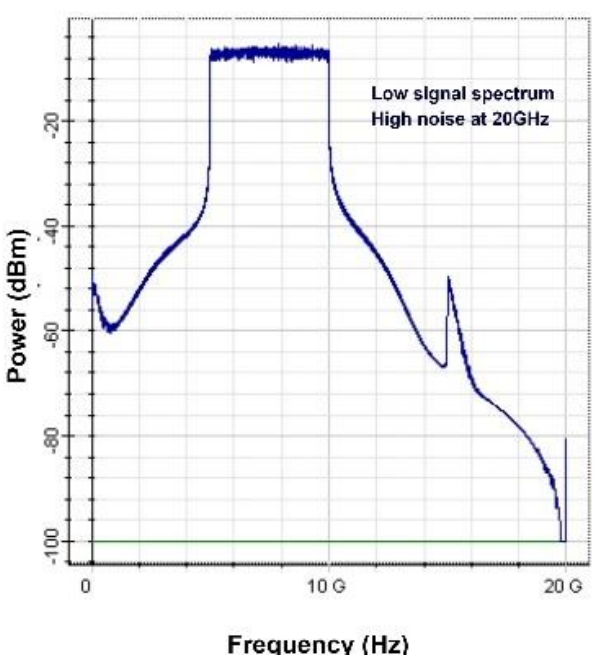

(c)

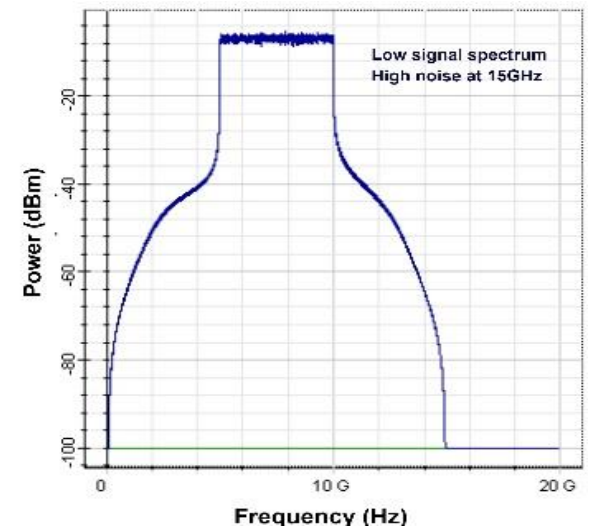

(b)

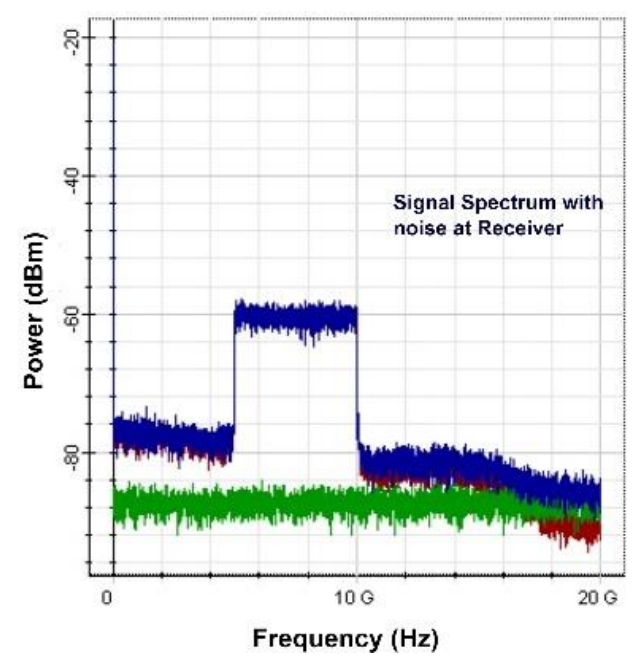

(d)

Figure 4. Signal spectrum at transmitter and receiver of 4 QAM-OFDM-FSO system, (a) OFDM signal spectrum, (b) roll-off factor 0.2 , (c) roll-off factor 1 , and (d) received signal spectrum

\subsection{Constellation and received power under various atmospheric losses}

The proposed system has been simulated for different ranges of FSO channels and attenuation of various weather conditions such as clear air, haze, and fog. Different deviations of constellation diagrams were produced. The larger the distance between the transmission aperture and the reception aperture of an FSO channel, the lower 
the received power $(\mathrm{dBm})$ and the higher the noise in the constellation visualizer. Figure 5 depicts the received power by the 4-QAM-OFDM technique under atmospheric attenuations $0.2 \mathrm{~dB} / \mathrm{km}$ for clear air, $4.2 \mathrm{~dB} / \mathrm{km}$ for haze, and $9.2 \mathrm{~dB} / \mathrm{km}$ for fog conditions. Good constellations were observed at $3 \mathrm{~km}, 1.5 \mathrm{~km}$, and $1 \mathrm{~km}$ for clear air, haze, and fog conditions. Comparisons of the constellation for 4-QAM and 4-PSK schemes are shown in Figure 6. It has been analyzed for $0.5 \mathrm{~km}$ and $0.7 \mathrm{~km}$ of the FSO channel under foggy conditions with attenuation $\alpha=22$ $\mathrm{dB} / \mathrm{km}$. Mapping constellation points in the 4-QAM input OFDM scheme is better than the 4-PSK input sequence. Also, power output values in each section of the complete model are compared in Table 3 for both input schemes.

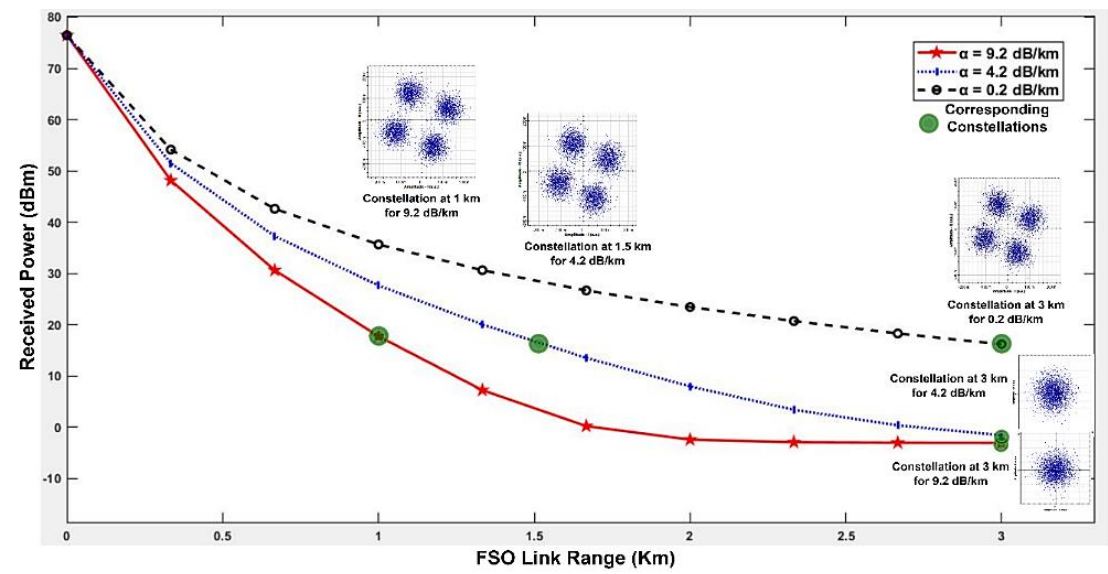

Figure 5. Received power and constellation comparison for clear air $(0.2 \mathrm{~dB} / \mathrm{km})$, haze $(4.2 \mathrm{~dB} / \mathrm{km})$ and fog $(9.2 \mathrm{~dB} / \mathrm{km})$

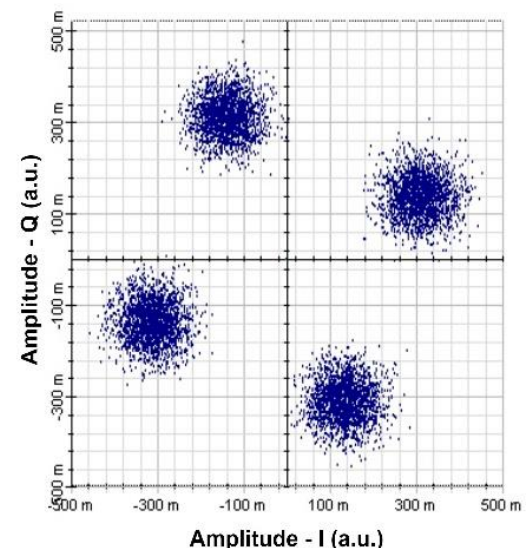

(a)

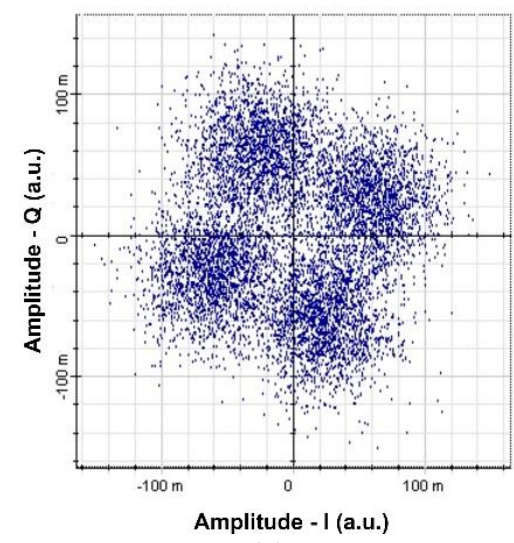

(c)

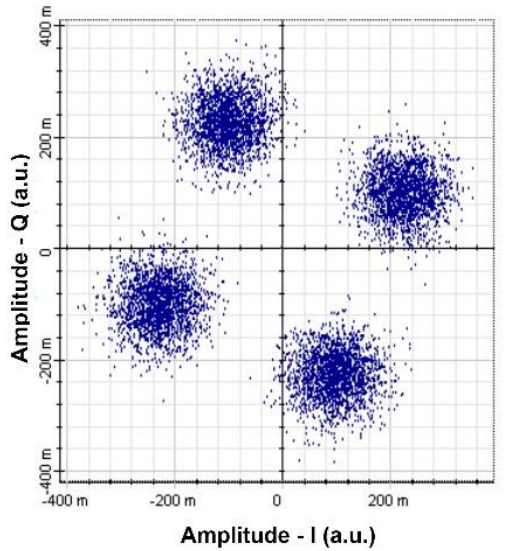

(b)

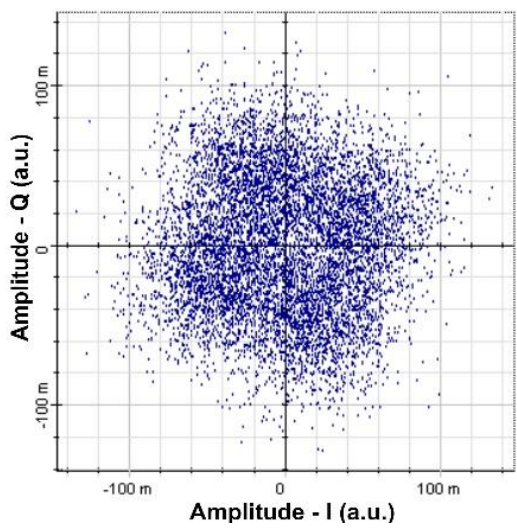

(d)

Figure 6. Heavy fog weather condition, $\alpha=22 \mathrm{~dB} / \mathrm{km}$, (a) $0.5 \mathrm{~km}$ (4-QAM), (b) $0.5 \mathrm{~km}$ (4-PSK), (c) $0.7 \mathrm{~km}$ (4-QAM), and (d) $0.7 \mathrm{~km}$ (4-PSK) 
Table 3. Power output at various stages of proposed model

\begin{tabular}{lcc}
\hline \multicolumn{1}{c}{ Output } & 4-QAM value $(\mathrm{dBm})$ & 4-PSK value $(\mathrm{dBm})$ \\
\hline OFDM & 57.082 & 54.013 \\
Quadrature Modulator & 17.070 & 14.060 \\
LiNb MZ modulator & -9.006 & -9.006 \\
Optical Amplifier after FSO channel & 5.104 & 5.105 \\
Direct detection at PIN Photodiode & -20.790 & -20.900 \\
System Receiver at the demodulator & 35.451 & 32.718 \\
\hline
\end{tabular}

\subsection{Constellation comparison (4-QAM and 4-PSK)}

The constellation diagrams are shown in Figures 5 and 6 clearly show various digital modulation performances in a given model. The proposed DD-OFDM based FSO model with 4-QAM digital input sequences shown in Figure 5 can achieve a $3 \mathrm{~km}$ FSO channel under the visibility of greater than $2 \mathrm{~km}$ having attenuation value $0.2 \mathrm{~dB} / \mathrm{km}$ for clear weather conditions, $1.5 \mathrm{~km}, 1 \mathrm{~km}$ with attenuations of $4.2 \mathrm{~dB} / \mathrm{km}, 9.2$ $\mathrm{dB} / \mathrm{km}$ respectively for thin, moderate fog conditions. Figures 6(a) and (b) are constellation points received for $0.5 \mathrm{~km}$ FSO link through 4-QAM and 4-PSK techniques under $22 \mathrm{~dB} / \mathrm{km}$ heavy fog conditions. Similarly, Figures 6(c) and (d) are shown for the $0.7 \mathrm{~km}$ FSO link.

\section{CONCLUSION}

FSO wireless optical channel performance analysis using DD-OFDM scheme with 4-QAM and 4-PSK input sequences is carried out, and simulated results are observed. According to the observations, the proposed model with 4-QAM input to OFDM performs better to reduce signal loss in free space under various weather conditions. Signal loss is considered in the atmosphere with attenuation values of $0.2 \mathrm{~dB} / \mathrm{km}$, $4.2 \mathrm{~dB} / \mathrm{km}, 9.2 \mathrm{~dB} / \mathrm{km}$, and $22 \mathrm{~dB} / \mathrm{km}$ for clear air, haze, and foggy conditions, and the FSO channel ranges varying from $0.5 \mathrm{~km}$ to $3 \mathrm{~km}$. Signal deterioration occurs as distance and attenuation increase, yet the model with 4-QAM input sequences to OFDM still produces the most efficient and cost-effective output than 4-PSK modulation. After being implemented using OFDM architecture, it was shown that the 4-QAM OFDM FSO method produced improved outputs at various conditions.

\section{REFERENCES}

[1] S. Addanki, I. S. Amiri, and P. Yupapin, "Review of optical fibers-introduction and applications in fiber lasers," Results in Physics, vol. 10, pp. 743-750, Sep. 2018, doi: 10.1016/j.rinp.2018.07.028.

[2] M. M. A. Eid, A. S. Seliem, A. N. Zaki Rashed, A. E. N. A. Mohammed, M. Y. Ali, and S. S. Abaza, "High modulated soliton power propagation interaction with optical fiber and optical wireless communication channels," Indonesian Journal of Electrical Engineering and Computer Science, vol. 21, no. 3, pp. 1575-1583, Mar. 2021, doi: 10.11591/ijeecs.v21.i3.pp1575-1583.

[3] A. K. Garg, V. Janyani, B. Batagelj, N. H. Zainol Abidin, and M. H. Abu Bakar, "Hybrid FSO/fiber optic link based reliable \& energy efficient WDM optical network architecture," Optical Fiber Technology, vol. 61, p. 102422, Jan. 2021, doi: 10.1016/j.yofte.2020.102422.

[4] M. Vinoth Kumar and V. Kumar, "Relative Investigation of Methods to Generate Millimeter Wave in Radio-Over-Fiber Communication," in Lecture Notes in Networks and Systems, vol. 106, Springer Singapore, 2020, pp. 567-574.

[5] M. Siddharth, S. Shah, and R. Swaminathan, "Outage analysis of adaptive combining scheme for hybrid FSO/RF communication," Feb. 2020, doi: 10.1109/NCC48643.2020.9056009.

[6] A. Mansour, R. Mesleh, and M. Abaza, "New challenges in wireless and free space optical communications," Optics and Lasers in Engineering, vol. 89, pp. 95-108, Feb. 2016, doi: 10.1016/j.optlaseng.2016.03.027.

[7] G. Z. and P. W., "Terrestrial Free-Space Optical Communications," in Mobile and Wireless Communications Network Layer and Circuit Level Design, InTech, 2010.

[8] R. Lange, B. Smutny, B. Wandernoth, R. Czichy, and D. Giggenbach, "142 km, 5.625 Gbps free-space optical link based on homodyne BPSK modulation," in Free-Space Laser Communication Technologies XVIII, Feb. 2006, vol. 6105, p. 61050A, doi: 10.1117/12.673749.

[9] S. G. Wilson, M. Brandt-Pearce, Q. Cao, and J. H. Leveque, "Free-space optical MIMO transmission with Q-ary PPM," IEEE Transactions on Communications, vol. 53, no. 8, pp. 1402-1412, Aug. 2005, doi: 10.1109/TCOMM.2005.852836.

[10] H. Sugiyama and K. Nosu, "MPPM: A Method for Improving the Band-Utilization Efficiency in Optical PPM," Journal of Lightwave Technology, vol. 7, no. 3, pp. 465-472, Mar. 1989, doi: 10.1109/50.16882.

[11] R. J. Green, "Comparison of pulse position modulation and pulse width modulation for application in optical communications," Optical Engineering, vol. 46, no. 6, p. 065001, Jun. 2007, doi: 10.1117/1.2746010.

[12] M. R. Abaza, N. A. Mohammed, and M. H. Aly, "BER performance of M-ary PPM free-space optical communications with channel fading," in 8th International Conference on High-Capacity Optical Networks and Emerging Technologies, HONET 2011, Dec. 2011, pp. 111-115, doi: 10.1109/HONET.2011.6149799.

[13] S. S. Muhammad, T. Javornik, I. Jelovčan, Z. Ghassemlooy, and E. Leitgeb, "Optical communications comparison of hard-decision and soft-decision channel coded M-ary PPM performance over free space optical links," European Transactions on Telecommunications, vol. 20, no. 8, pp. 746-757, Dec. 2009, doi: 10.1002/ett.1343.

[14] S. Sharma, A. S. Madhukumar, and R. Swaminathan, "Mimo hybrid fso/rf system over generalized fading channels," IEEE Transactions on Vehicular Technology, vol. 70, no. 11, pp. 11565-11581, Nov. 2021, doi: 10.1109/TVT.2021.3111401. 
[15] M. Vinothkumar and V. Kumar, "Simulation and Analysis of 5G Wireless mm-Wave Modulation Technique for High Capacity Communication System," in Trends in Mathematics, Springer International Publishing, 2021, pp. 107-111.

[16] M. Safari and M. Uysal, "Relay-assisted free-space optical communication," IEEE Transactions on Wireless Communications, vol. 7, no. 12, pp. 5441-5449, Dec. 2008, doi: 10.1109/T-WC.2008.071352.

[17] G. A. Pethunachiyar and B. Sankaragomathi, "Fast and accurate primary user detection with machine learning techniques for cognitive radio networks," Indonesian Journal of Electrical Engineering and Computer Science, vol. 21, no. 1, pp. 472-478, Jan. 2021, doi: 10.11591/ijeecs.v21.i1.pp472-478.

[18] M. A. Esmail, W. S. Saif, A. M. Ragheb, and S. A. Alshebeili, "Free space optic channel monitoring using machine learning," Optics Express, vol. 29, no. 7, p. 10967, Mar. 2021, doi: 10.1364/oe.416777.

[19] M. Rungruanganukul and T. Siriborvornratanakul, "Deep Learning Based Gesture Classification for Hand Physical Therapy Interactive Program," in Lecture Notes in Computer Science (including subseries Lecture Notes in Artificial Intelligence and Lecture Notes in Bioinformatics), vol. 12198 LNCS, Springer International Publishing, 2020, pp. 349-358.

[20] C. Kerdvibulvech and L. (Luke) Chen, "The Power of Augmented Reality and Artificial Intelligence During the Covid-19 Outbreak," in Lecture Notes in Computer Science (including subseries Lecture Notes in Artificial Intelligence and Lecture Notes in Bioinformatics), vol. 12424 LNCS, Springer International Publishing, 2020, pp. 467-476.

[21] L. Darwesh and N. S. Kopeika, "Deep Learning for Improving Performance of OOK Modulation over FSO Turbulent Channels," IEEE Access, vol. 8, pp. 155275-155284, 2020, doi: 10.1109/ACCESS.2020.3019113.

[22] F. Aveta, H. H. Refai, and P. G. Lopresti, "Cognitive Multi-Point Free Space Optical Communication: Real-Time Users Discovery Using Unsupervised Machine Learning,” IEEE Access, vol. 8, pp. 207575-207588, 2020, doi: 10.1109/ACCESS.2020.3038624.

[23] F. Khair, H. P. Fakhriy, I. W. Mustika, B. Setiyanto, and S. M. Idrus, "Modeling and simulation of OFDM scheme for radio over fiber (RoF)," in ICITACEE 2015 - 2nd International Conference on Information Technology, Computer, and Electrical Engineering: Green Technology Strengthening in Information Technology, Electrical and Computer Engineering Implementation, Proceedings, Oct. 2016, pp. 376-381, doi: 10.1109/ICITACEE.2015.7437833.

[24] A. H. Ali, H. J. Alhamdane, and B. S. Hassen, "Design analysis and performance evaluation of the WDM integration with COOFDM system for radio over fiber system," Indonesian Journal of Electrical Engineering and Computer Science, vol. 15, no. 2, pp. 870-878, Aug. 2019, doi: 10.11591/ijeecs.v15.i2.pp870-878.

[25] I. I. Kim, B. McArthur, and E. J. Korevaar, "Comparison of laser beam propagation at $785 \mathrm{~nm}$ and $1550 \mathrm{~nm}$ in fog and haze for optical wireless communications," in Optical Wireless Communications III, Feb. 2001, vol. 4214, pp. 26-37, doi: $10.1117 / 12.417512$

[26] M. A. Al-Habash, "Mathematical model for the irradiance probability density function of a laser beam propagating through turbulent media," Optical Engineering, vol. 40, no. 8, p. 1554, Aug. 2001, doi: 10.1117/1.1386641.

[27] N. S. Alagha and P. Kabal, "Generalized raised-cosine filters," IEEE Transactions on Communications, vol. 47, no. 7, pp. 989997, Jul. 1999, doi: 10.1109/26.774849.

[28] L. C. Andrews, R. L. Phillips, and C. Y. Young, Laser Beam Scintillation with Applications. SPIE, 2009.

[29] M. Uysal, S. M. Navidpour, and J. Li, "Error rate performance of coded free-space optical links over strong turbulence channels," IEEE Communications Letters, vol. 8, no. 10, pp. 635-637, Oct. 2004, doi: 10.1109/LCOMM.2004.835306.

[30] E. Bayaki, R. Schober, and R. K. Mallik, "Performance analysis of free-space optical systems in gamma-gamma fading," in GLOBECOM - IEEE Global Telecommunications Conference, 2008, pp. 2859-2864, doi: 10.1109/GLOCOM.2008.ECP.548.

[31] A. García-Zambrana, C. Castillo-Vázquez, and B. Castillo-Vázquez, "On the capacity of FSO links over gamma-gamma atmospheric turbulence channels using OOK signaling," Eurasip Journal on Wireless Communications and Networking, vol. 2010, no. 1, May 2010, doi: 10.1155/2010/127657.

\section{BIOGRAPHIES OF AUTHORS}
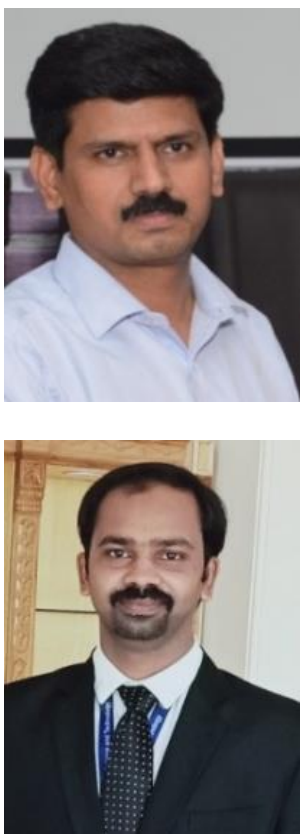

Dr. Vinod Kumar (iD) SC P received his B. E. degree in Electronics Engg. from Nagpur University, Nagpur, India, and M. Tech, Ph.D. degree from the Department of Electronics Engineering, Indian Institute of Technology (BHU) Varanasi, India. Presently, he is working as an Associate Professor in the Department of ECE, SRM Institute of Science and Technology, Delhi-NCR, Ghaziabad, India. He has published several papers in IEEE Sensors, IEEE Transaction, AIP, Elsevier, Springer, etc. His research interest includes high power microwave devices and circuits, Optoelectronic devices, Wireless Sensor Networks, MOS sensors, fabrication \& characterization of thin films devices, fabrication and characterization of MOS devices and plasma-processed devices. He can be contacted at email: vinodkur1@srmist.edu.in.

M. Vinoth Kumar (D) 81 SC $P$ is currently pursuing his Ph.D. degree at the Department of Electronics and Communication Engineering, SRM Institute of Science and Technology, Delhi-NCR Campus, India. He received his M.Tech. VLSI design and Embedded Systems from B. S. Abdur Rahman University, Chennai, Tamil Nadu, India, in 2013 and B.E. Electronics and Instrumentation Engineering from Anna University, Tamil Nadu, India, in 2009. His research interests are optical fiber communication networks, free-space optical communication systems, photonics, and radio-over-fiber. He can be contacted at email: vinothkm@srmist.edu.in. 